\title{
BMJ Open Nationwide seroprevalence of antibodies to SARS-CoV-2 in asymptomatic population in South Korea: a cross-sectional study
}

\author{
Eun-Hee Nah (D) , ${ }^{1}$ Seon Cho, ${ }^{1}$ Hyeran Park, ${ }^{1}$ Inhwan Hwang, ${ }^{1}$ Han-Ik Cho ${ }^{2}$
}

To cite: Nah E-H, Cho S,

Park $\mathrm{H}$, et al. Nationwide seroprevalence of antibodies to SARS-CoV-2 in asymptomatic population in South Korea: a cross-sectional study. BMJ Open 2021;11:e049837. doi:10.1136/ bmjopen-2021-049837

- Prepublication history for this paper is available online. To view these files, please visit the journal online (http://dx.doi. org/10.1136/bmjopen-2021049837).

Received 03 February 2021 Revised 25 March 2021 Accepted 12 April 2021

Check for updates

(C) Author(s) (or their employer(s)) 2021. Re-use permitted under CC BY-NC. No commercial re-use. See rights and permissions. Published by BMJ.

${ }^{1}$ Health Promotion Research Institute, Korea Association of Health Promotion, Seoul, Republic of Korea

${ }^{2}$ MEDIcheck LAB, Korea

Association of Health Promotion, Seoul, Republic of Korea

Correspondence to

Dr Eun-Hee Nah;

cellonah@hanmail.net

\section{ABSTRACT}

Objective Asymptomatic active infection might be an important contributor to the COVID-19 outbreak. Serological tests can assess the extent of exposure and herd immunity to COVID-19 in general populations. This study aimed to estimate the nationwide seroprevalence of SARS-CoV-2 antibodies according to age, sex and clinical status in South Korea.

Design, setting and participants This cross-sectional study randomly selected health examinees who underwent health check-up at 16 health promotion centres in 13 Korean cities across the country between late September and early December 2020. Residual serum samples were obtained from 4085 subjects (2014 men and 2071 women). Antibodies to SARS-CoV-2 were measured by electrochemiluminescence immunoassay using Elecsys Anti-SARS-CoV-2 (Roche Elecsys, Mannheim, Germany). Primary and secondary outcome measures Fisher's exact test was used to compare the seroprevalence according to sex, age group and region. The relative risks of being seropositive according to the characteristics of the study subjects were analysed using logistic regression analysis.

Results The overall seroprevalence of anti-SARS-CoV-2 was $0.39 \%$ ( $95 \% \mathrm{Cl}=0.20 \%$ to $0.58 \%): 0.30 \%$ (95\% $\mathrm{Cl}=0.06 \%$ to $0.54 \%)$ for men and $0.48 \%(95 \% \mathrm{Cl}=0.18 \%$ to $0.78 \%$ ) for women. The rate of anti-SARS-CoV-2 positivity varied significantly between different regions of Korea ( $p=0.003$ ), but not with age group, sex, or the statuses of obesity, diabetes, hypertension or smoking. Conclusions Most of the Korean population is still immunologically vulnerable to SARS-CoV-2, but the seroprevalence has increased relative to that found in studies performed prior to September 2020 in Korea.

\section{INTRODUCTION}

The COVID-19 outbreak has continued sporadically in Korea since the first case was detected on 20 January 2020 in Korea. ${ }^{1}$ This is despite the stringent Korean interventions against COVID-19 consisting of massive testing using the reverse-transcription PCR (RT-PCR), contact tracing and quarantining, which have been considered a model for controlling the COVID-19 pandemic.
Strengths and limitations of this study

The strength of this study lies in enrolling subjects from 13 cities all over the country.

- This study showed the seroprevalence of antiSARS-CoV-2 in other times of the different waves of the COVID-19 pandemic.

- This study provides not only nationwide but also regional seroprevalence of the anti-SARS-CoV-2 in South Korea.

- Selection bias associated with the reasons for undergoing health check-ups might have been present.

- The sample size is not large enough to analyse the relative risks of being seropositive according to the characteristics of the study subjects.

COVID-19 is currently mainly detected in symptomatic individuals who have had close contact with confirmed patients and those with a history of travel to affected regions or entrants from abroad. Asymptomatic individuals without a history of close contact with confirmed patients are generally not screened in Korea. SARS-CoV-2 is a highly contagious virus. $^{2}$ That is also detected in asymptomatic individuals, which means that subclinical active infection might be an important contributor to the COVID-19 pandemic. ${ }^{3}$

The epidemiological significance of patients with asymptomatic and mild COVID-19 has been emphasised since these patients shed a considerable viral load without noticeable symptoms and could remain undetected. ${ }^{4}$ COVID-19 is diagnosed based on a viral RNA test using the RT-PCR. ${ }^{5}$ This is a sensitive method for detecting SARS-CoV-2, but large numbers of subclinical and asymptomatic infected individuals might remain undetected by symptom-based screening strategies. ${ }^{6}$ Seroepidemiological studies can reveal the prevalence of asymptomatic or subclinical infection in the community. ${ }^{7}$ Moreover, surveillance of antibody seropositivity can 
reveal the cumulative prevalence of SARS-CoV-2 infection and herd immunity to COVID-19 in both vulnerable and general populations. ${ }^{89}$

Antibodies, particularly IgG, are likely to persist after the viral infection has cleared, and serological tests can identify individuals exposed to the virus and so assess the extent of population exposure. A few studies have investigated the seroprevalence in Korea, ${ }^{10}{ }^{11}$ but they have focused on restricted geographical areas and were conducted during early-to-mid-2020. Considering that the seroepidemiology may change as the pandemic progresses, seroepidemiological studies should be performed repeatedly on a nationwide basis. Therefore, the present study aimed to estimate the nationwide seroprevalence and characteristics of SARS-CoV-2 infection in South Korea.

\section{METHODS}

\section{Study design and participants}

This cross-sectional study randomly selected health examinees who underwent health check-ups at 16 health promotion centres in 13 Korean cities across the country between late September 2020 and early December 2020. Residual serum samples were obtained for the study. The 16 health promotion centres belong to the Korea Association of Health Promotion, with 3 in Seoul, 2 in Daegu, and 1 in each of Busan, Ulsan, Changwon, Incheon, Jeonju, Kwangju, Daejeon, Suwon, Chuncheon, Chungju and Jeju. Korea has a national health insurance system (NHIS) that covers the entire population of South Korea and provides biennial medical examinations. These 16 health promotion centres, which are located across the nation, perform about $10 \%$ of the health check-ups that are provided by the NHIS in South Korea.

We calculated the required sample size with the following formula ${ }^{12}: n=\frac{Z^{2} P(1-P)}{d^{2}}$

Where $n$ is the sample size, $Z$ is the statistic corresponding to level of confidence, $P$ is expected prevalence and $d$ is precision. We assumed a nationwide seroprevalence of SARS-CoV-2 antibody of $1.5 \%{ }^{13}$ In our study, $Z$ is $1.96, P$ is 0.015 and $d$ is 0.004 (one-fourth of expected seroprevalence). We randomly selected subjects from health examinees using stratification by region and sex. Required subjects were allocated to health promotion centres proportional to regional population size where centres were located and stratified randomisation underwent with regard to sex.

Subjects who had COVID-19-related symptoms or who had a history of return from abroad or contact with confirmed COVID-19 patients within 2 weeks were excluded. Those who had a history of malignancy, stroke or myocardial infarction were also excluded. The selfreported personal medical history and lifestyle information (smoking, alcohol and exercise) were obtained at the time of health check-ups. The following demographic characteristics and results of biochemical tests were extracted electronically: age, sex, body mass index (BMI), waist circumference, blood pressure, laboratory results (complete blood count (CBC), fasting blood sugar, lipids, creatinine), and medical comorbidities (hypertension, diabetes and cardiovascular diseases).

\section{Laboratory measurements}

Antibodies to SARS-CoV-2 (including $\operatorname{IgG}$ ) were measured by electrochemiluminescence immunoassay (ECLIA) using Elecsys Anti-SARS-CoV-2 (Roche Elecsys, Mannheim, Germany) according to the manufacturer's protocol. That assay uses a recombinant protein representing the nucleocapsid protein of SARS-CoV-2 with a double-antigen sandwich assay. The clinical sensitivity and specificity of the assay were reported as $99.5 \%$ and $99.8 \%$, respectively, in PCR-confirmed SARS-CoV-2 cases when tested at $\geq 14$ days post-PCR confirmation. ${ }^{14}$ The intra-assay and total variances were $1.8 \%$ and $2.0 \%$, respectively. A cut-off index (COI, signal sample/cutoff) of $\geq 1.0$ was considered to indicate anti-SARS-CoV-2 positivity. The CBC and biochemical parameters were measured using the Sysmex XE-2100D analyser (Sysmex, Kobe, Japan) and the Hitachi 7600 analyser (Hitachi, Tokyo, Japan), respectively.

\section{Statistical analyses}

Statistical analyses were performed using SAS V.9.4 (SAS Institute). Data are presented as mean $\pm \mathrm{SD}$ or frequency (percentage) values. Prevalence and 95\% CI values were calculated. Fisher's exact test was used to compare the prevalence of seroprevalence according to sex, age group and region. Differences in seroprevalence among subgroups and the relative risk of being seropositive according to subject characteristics were analysed using Fisher's exact tests and logistic regression analysis, respectively. Differences in subjects with anti-SARS-CoV-2 positivity according to the history of recovering from COVID-19 were analysed using Mann-Whitney and Fisher's exact test. A p value of $<0.05$ was considered statistically significant.

\section{Patient and public involvement}

Patients were not involved in the recruitment to and conduct of the study. Results will be disseminated to study participants through annual information events.

\section{RESULTS}

\section{Characteristics of study subjects}

The characteristics of the 4085 (2014 men and 2071 women) study subjects are listed in table 1 . The subjects were aged $52.29 \pm 11.66$ years (range $18-86$ years). The age distribution was as follows: $129(3.2 \%)$ aged $<30$ years, $450(11.0 \%)$ aged $30-39$ years, $978(23.9 \%)$ aged $40-49$ years, $1260(30.8 \%)$ aged $50-59$ years, $1034(25.3 \%)$ aged $60-69$ years, and $234(5.7 \%)$ aged $\geq 70$ years. There were no differences between subjects with anti-SARS-CoV-2 
Table 1 Characteristics of the study subjects according to the presence of anti-SARS-CoV-2 antibodies

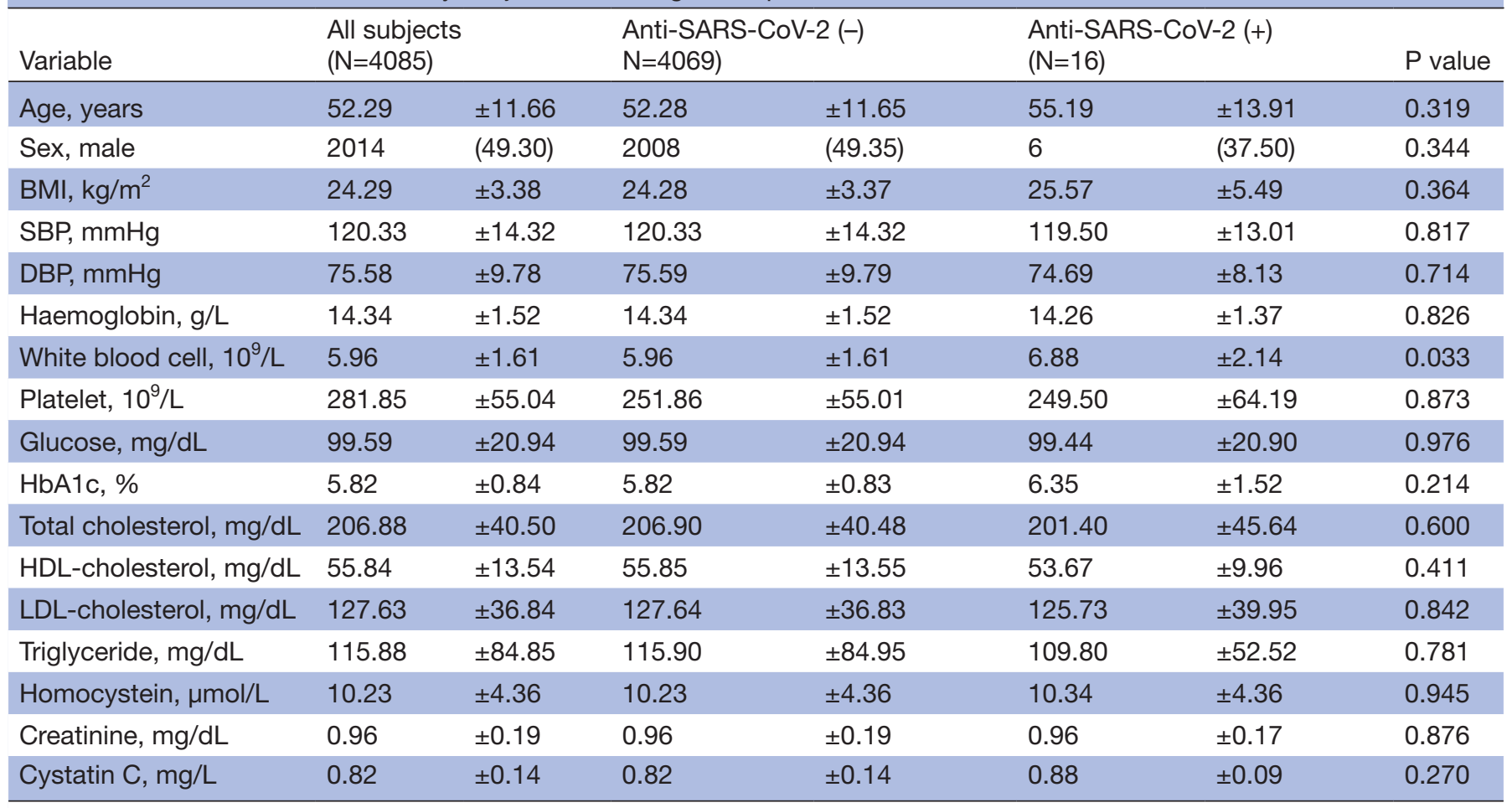

Data are mean \pm SD or $N(\%)$ values. $P$ values are from $t$-tests and $X^{2}$ tests.

BMI, body mass index; DBP, diastolic blood pressure; HbA1c, glycated haemoglobin; HDL, high-density lipoprotein; LDL, low-density lipoprotein; SBP, systolic blood pressure.

positivity and negativity in BMI, blood pressure, CBC (except white blood cell), blood lipids or creatinine (table 1).

\section{Seroprevalence according to age, sex and characteristics of study subjects}

The 4085 subjects included 16 anti-SARS-CoV-2-positive cases, of which 6 had previously recovered from COVID19. The overall seroprevalence of anti-SARS-CoV-2 was $0.39 \%$ (95\% CI $=0.20 \%$ to $0.58 \%): 0.30 \%(95 \% \mathrm{CI}=0.06 \%$ to $0.54 \%$ ) for men and $0.48 \%$ ( $95 \% \mathrm{CI}=0.18 \%$ to $0.78 \%$ ) for women. The seroprevalence did not differ significantly with sex, age group, or statuses of obesity, diabetes, hypertension or smoking (data not shown).

\section{Seroprevalence of anti-SARS-CoV-2 by region}

The anti-SARS-CoV-2 positivity rate varied between different regions $(\mathrm{p}=0.003)$ (data not shown). Figure 1 shows the seroprevalence of anti-SARS-CoV-2 at the sampling site of the health promotion centres. The seroprevalence ranged from $0.26 \%$ to $0.45 \%$ in three centres in the Seoul area, and from $0.97 \%$ to $2.63 \%$ in Daegu and its neighbouring regions, which was the epicentre of the COVID-19 outbreak in February 2020. The seroprevalence of anti-SARS-CoV-2 was $1.79 \%$ in Jeju, but this was a single seropositive case among only 56 subjects.
Characteristics of anti-SARS-CoV-2-positive subjects according to the history of recovering from COVID-19

Six of the 16 seropositive cases had a history of recovering from COVID-19, 4 of which (with COI values of 41-125) were diagnosed using PCR tests in Daegu and its neighbouring areas between February and March, 2020, with 1

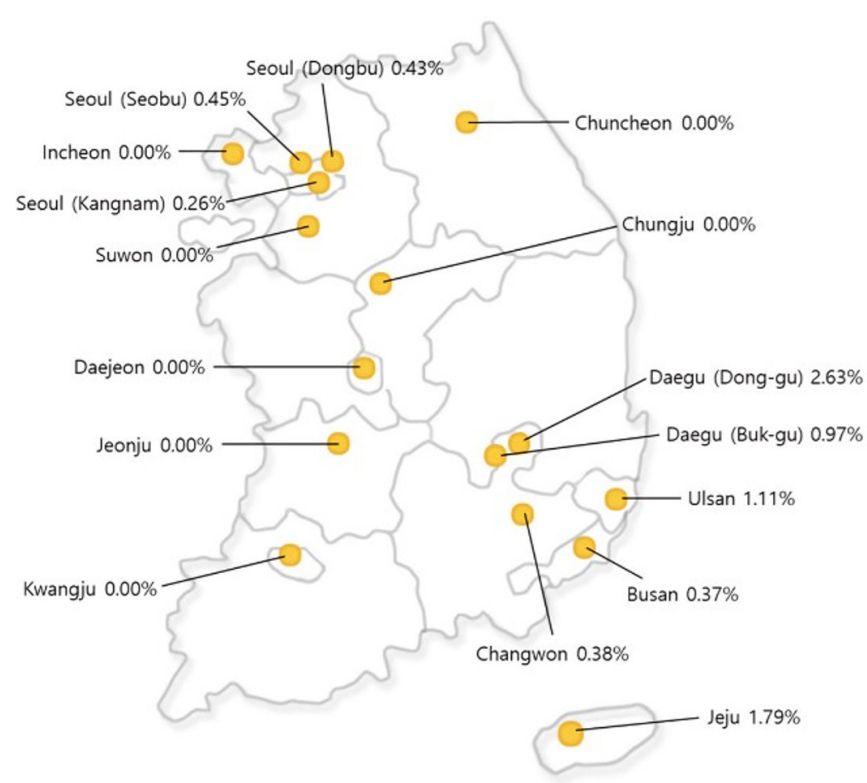

Figure 1 Map showing anti-SARS-CoV-2 seroprevalence rates at the sampling sites of the health promotion centres. 


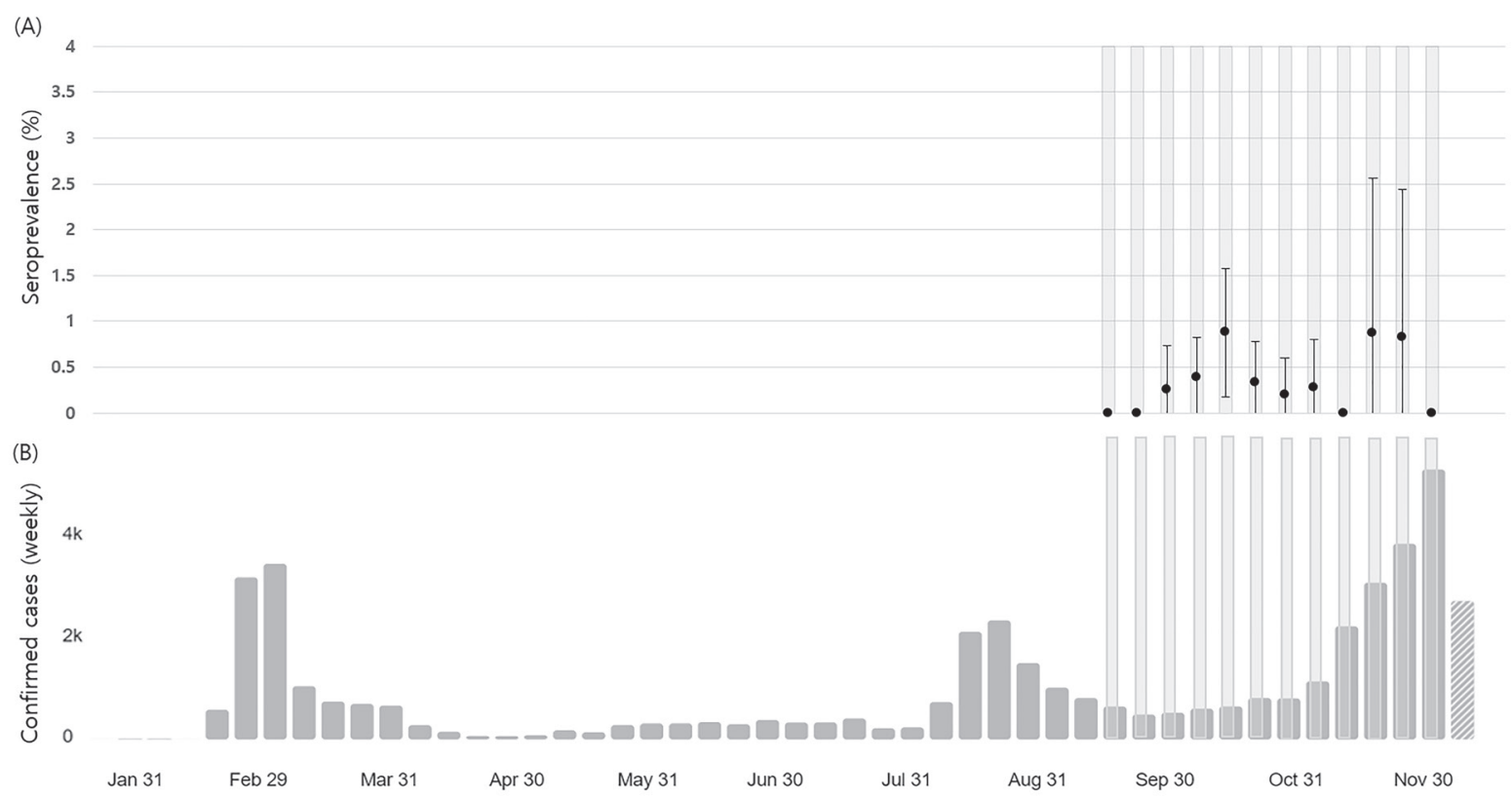

Figure 2 Seroprevalence and 95\% Cls for each week of the study (A), weekly confirmed COVID-19 cases in South Korea cited from the WHO COVID-19 Dashboard (B). Yellow shading shows the sampling periods for each week in this study.

case in each of Seoul and Jeju diagnosed using PCR test in mid-September and mid-August, respectively. The median COI value of positive samples did not differ significantly between subjects with and without a history of recovering from COVID-19 (51.6 vs 7.4, $\mathrm{p}=0.104$ ) (data not shown).

\section{Seroprevalence and $\mathbf{9 5 \%}$ Cls for each week of the study}

Figure 2A,B shows the seroprevalence and 95\% CIs during each week of the study and the weekly confirmed COVID-19 cases in South Korea. There were four seropositive cases from the three Seoul centres during the 4 weeks on November, which was the beginning of the third wave of the COVID-19 outbreak in Seoul and its neighbouring areas.

\section{DISCUSSION}

This study has revealed the seroprevalence of antiSARS-CoV-2 and its characteristics from the end of the second wave to the beginning of the third wave of the COVID-19 pandemic in South Korea. The seroprevalence was $0.39 \%$ overall and varied in different regions. Antibodies (including IgG) can remain for more than 7 months after infection in individuals who have recovered from COVID-19. This finding suggests that most of the Korean population is still immunologically vulnerable to SARS-CoV-2 despite the seroprevalence being higher than that reported prior to September 2020 in Korea.

The seroprevalence of anti-SARS-CoV-2 antibodies varies in countries, study populations, times of sampling, laboratory testing methods and incidence rates of COVID-19 at the sampling sites (table 2). A cross-sectional study of 10 sites in the USA conducted between 23 March and 12 May 2020 estimated seroprevalence rates ranged from $1.0 \%$ to $6.9 \% .{ }^{15}$ The specimens in that study were collected for clinical purposes from persons seeking healthcare, who might not be representative of the general population. In a population-based study conducted in Spain from 27 April to 11 May 2020, the seroprevalence was $5.0 \%$ by the point-of-care test and $4.6 \%$ by immunoassay. That study found substantial geographical variability in the prevalence, being $>10 \%$ around Madrid and $<3 \%$ in coastal areas. ${ }^{16} \mathrm{~A}$ cross-sectional survey in Greece performed from March and April 2020 found a seroprevalence of $0.36 \%$ among 6586 leftover sera collected from a nationwide laboratory network. ${ }^{17}$ In two nationwide serological household surveys in Brazil, the seroprevalence rates in the first survey (May 14-21) and second survey (June 4-7) were $1.9 \%$ and $3.1 \%$, respectively, ${ }^{18}$ when Brazil was a global hot spot for COVID-19. These surveys showed that the prevalence is higher among those living in crowded conditions and with an indigenous ancestry and a low socioeconomic status. The seroprevalence in Wuhan (the epicentre of the pandemic in China) from 9 March to 10 April 2020 varied between $3.2 \%$ and $3.8 \%$ in different subcohorts, ${ }^{19}$ and progressively decreased in other cities that were further from Wuhan.

Seroprevalence studies performed in Daegu and southwestern Seoul, and involving the Korean National Health and Nutritional Examination Survey (NHANES) have used different assays, sampling times and subject 


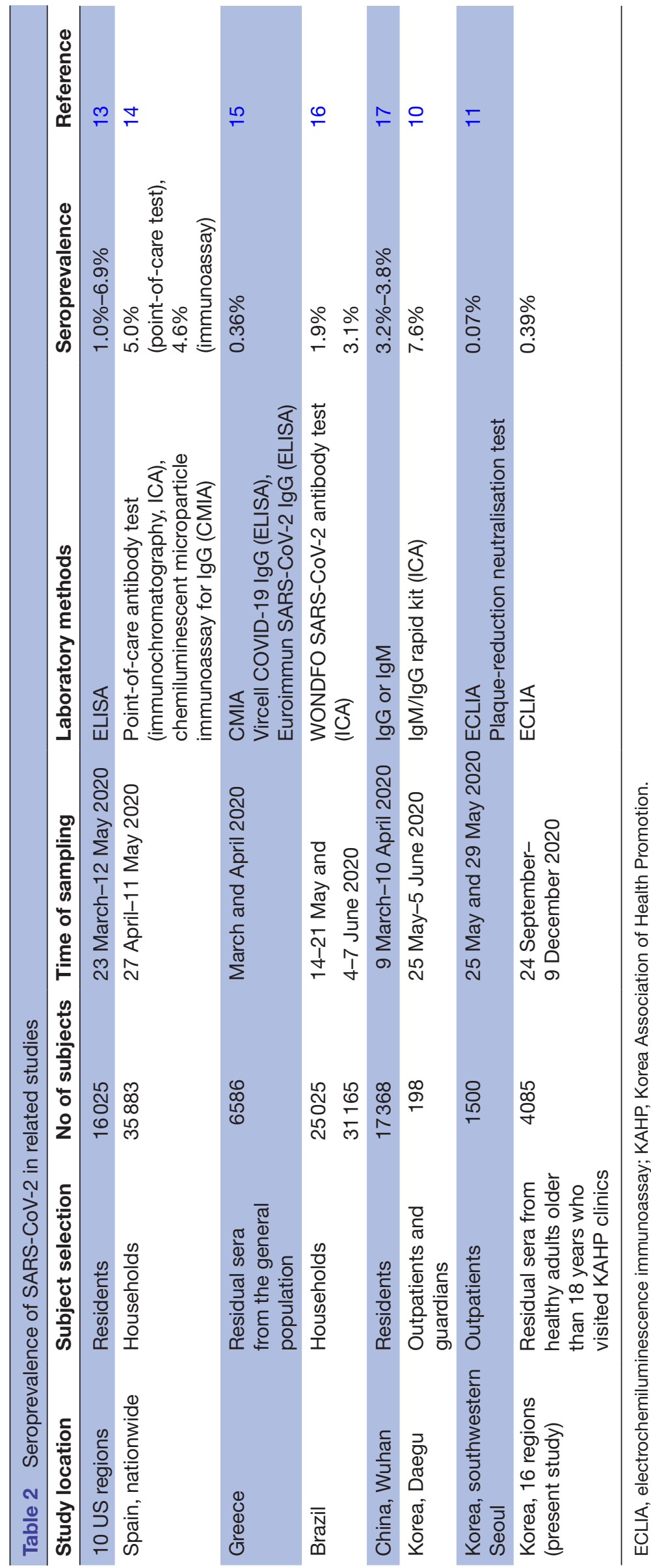

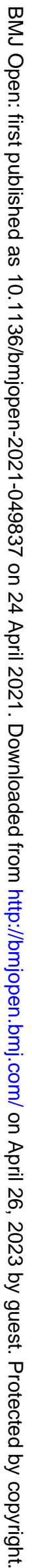


selection methods. The study in Daegu (the epicentre of COVID-19 in late February in Korea) conducted from 25 May to 5 June 2020 estimated a seroprevalence of $7.6 \% .^{10}$ However, the serological tests were conducted in 103 outpatients and 95 guardians with SARS-CoV-2 using the rapid lateral flow assay, which is controversial due to its heterogeneous and inconsistent results. ${ }^{20}$ Moreover, the study subjects comprised outpatients and their guardians at a single hospital, limiting the generalisability of the results. A study in southwestern Seoul conducted between 25 May and 29 May 2020 found one seropositive case among 1500 university hospital outpatients, giving a seroprevalence of $0.07 \% .{ }^{11} \mathrm{~A}$ study that used serum samples of participants who had participated twice in NHANES in 13 regions of South Korea, from 21 April to 16 June and from 10 June to 13 August 2020, found no seropositive case among 1555 samples and one seropositive case among 1440 samples, respectively. ${ }^{21}$

Our nationwide study in South Korea estimated a seroprevalence of $0.39 \%$. If the six cases that had previously recovered from COVID-19 were excluded from the 16 seropositive cases, the seroprevalence was $0.25 \%$. Although this rate is higher than those for the NHANES surveys in Korea, this relatively low prevalence may reflect that the reported incidence and mortality of COVID-19 were relatively low in Korea during the study period. The COVID-19 pandemic has been controlled in Korea by implementing public health measures such as social distancing, contact tracing, active testing and quarantining. The present study was conducted between the last week of September and the first week of December, which was from the end of the second wave to the beginning of the third wave of the COVID-19 pandemic in South Korea.

Our study found substantial regional variability in the prevalence, being higher in Seoul, Daegu and their neighbouring regions. Seoul is a densely populated city that has been a hot spot of COVID-19, while Daegu (the fourth largest city in Korea) experienced an explosive increase in the number of patients with COVID-19 in late February. Most of the seropositive cases in our study were found in these regions. We found that the seroprevalence did not vary with age or sex, whereas several nationwide, population-based seroepidemiological studies ${ }^{15} 1618$ found that seroprevalence rates differed by region but not by sex. However, the association between seroprevalence and age has varied between studies. One study of 10 sites in the USA found no difference in seroprevalence between age groups,${ }^{15}$ whereas Hallal et $a l^{18}$ observed that the prevalence was higher in participants aged 20-59 years, and Stringhini $e t a l^{22}$ found that it was significantly lower in individuals aged $5-9$ years and $>65$ years than in those aged $20-49$ years.

The early detection of SARS-CoV-2 infection is a crucial intervention for controlling the virus transmission. During the course of a pandemic, identifying individuals with asymptomatic infection is one of the main challenges because they could be a major source of transmission. The RT-PCR test is the reference method for diagnosing SARS-CoV-2 infection, but false-negative results are possible due to improper handling of nucleic acid samples and inadequate sampling. ${ }^{23}$ In addition, SARS-CoV-2 viral RNA becomes almost undetectable 14 days after illness onset. ${ }^{24}$ Immunoassays may be able to reduce the number of undiagnosed cases.

The timing, titre, and duration of anti-SARS-CoV-2 antibodies and the optimal protection against the COVID-19 remain to be established. The timing of the development of SARS-CoV-2-specific antibodies is variable: reactive IgA, IgM and IgG antibodies have been detected 1 day after symptom onset, ${ }^{25}$ but the median development times for total antibodies, IgM, and IgG have been estimated as 11,12 and 14 days, respectively. ${ }^{26}{ }^{27}$ One study found no specific chronological order in terms of IgM and IgG seroconversion. ${ }^{28}$ In our study, four cases were previously diagnosed with PCR tests and recovered in Daegu and its neighbouring areas between February and March 2020. This suggests that antibodies (including $\operatorname{IgG}$ ) can remain in individuals who recover from COVID-19 for more than 7 months after infection.

Our study had some limitations. First, selection bias associated with the reasons for undergoing health check-ups might have been present. Subjects were voluntary health check-up examinees rather than general population. They may be health conscious and risk averse, so that the seroprevalence was potentially underestimated. However, our study subjects comprised health examinees from 16 health promotion centres in 13 cities across Korea that routinely perform NHIS health check-ups. This study population was expected to be representative of the general population of Korea, without restriction to specific classes or areas. Moreover, since we excluded those who had recent symptomatic illness or who had a history of return from abroad or contact with patients with confirmed COVID-19 within 2 weeks were excluded from health check-ups, there was rare possibility of specimens drawn from patients seeking care for suspected COVID-19 symptoms, potentially biasing the results. Second, sample size is not large enough to estimate the nationwide seroprevalence of anti-SARS-CoV-2 antibody and analyse the relative risks of being seropositive according to the characteristics of the study subjects because the seroprevalence was much lower than the assumed seroprevalence of $1.5 \%$ in the sample size calculation. Third, since the ECLIA may be affected by cross-reactivity with antibodies to other common cold coronaviruses, false-positive results for SARS-CoV-2 were possible, potentially leading to overestimation of the actual seroprevalence in the low prevalence setting of South Korea.

In conclusion, this study has provided the nationwide and regional seroprevalence rates of SARS-CoV-2 from the end of the second wave to the beginning of the third wave of the COVID-19 pandemic in South Korea. The seroprevalence varies across regions but is lower than those in most other countries and insufficient to provide herd immunity. However, the prevalence was higher than 
that found studies conducted prior to September 2020 in Korea. New cases of COVID-19 continuously appear even under the stringent interventions adopted in Korea. The present findings suggest that asymptomatic seropositive individuals contribute to virus transmission. The strategy of applying PCR tests to suspected patients and quarantining should be continued. In addition, screening for SARS-CoV-2 using anti-SARS-CoV-2 antibody tests in the asymptomatic populations could be considered in risk region with high incidence rate.

Acknowledgements The authors would like to thank all participants who participated in this study and all staff members of health promotion centres and MEDIcheck LAB, Korea Association of Health Promotion, Seoul, Korea.

Contributors All of the authors participated in designing this study. SC, HP and IH performed data collection. SC undertook the statistical analyses. E-HN, SC and $\mathrm{H}-\mathrm{IC}$ analysed and interpreted the data. E-HN wrote the first draft of the manuscript, which was reviewed by all of the other authors, who also provided further contributions and suggestions.

Funding This study was supported by Roche Diagnostics through Investigator Initiated Study, which provided reagents for testing anti-SARS-CoV-2 antibodies.

Map disclaimer The depiction of boundaries on this map does not imply the expression of any opinion whatsoever on the part of BMJ (or any member of its group) concerning the legal status of any country, territory, jurisdiction or area or of its authorities. This map is provided without any warranty of any kind, either express or implied.

Competing interests None declared.

Patient consent for publication Not required.

Ethics approval This study was approved by the Institutional Review Board of the Korea Association of Health Promotion (approval no. 130 750-202008-HR-013).

Provenance and peer review Not commissioned; externally peer reviewed.

Data availability statement № data are available.

Open access This is an open access article distributed in accordance with the Creative Commons Attribution Non Commercial (CC BY-NC 4.0) license, which permits others to distribute, remix, adapt, build upon this work non-commercially, and license their derivative works on different terms, provided the original work is properly cited, appropriate credit is given, any changes made indicated, and the use is non-commercial. See: http://creativecommons.org/licenses/by-nc/4.0/.

ORCID iD

Eun-Hee Nah http://orcid.org/0000-0003-0637-4364

\section{REFERENCES}

1 Kim JY, Choe PG, Oh Y, et al. The first case of 2019 novel coronavirus pneumonia imported into Korea from Wuhan, China: implication for infection prevention and control measures. J Korean Med Sci 2020;35:e61.

2 Sanche S, Lin YT, Xu C, et al. High contagiousness and rapid spread of severe acute respiratory syndrome coronavirus 2. Emerg Infect Dis 2020;26:1470-7.

3 Bai Y, Yao L, Wei T, et al. Presumed asymptomatic carrier transmission of COVID-19. JAMA 2020;323:1406-7.

4 Huff HV, Singh A. Asymptomatic transmission during the coronavirus disease 2019 pandemic and implications for public health strategies. Clin Infect Dis 2020;71:ciaa654:2752-6.

5 Ahmed SF, Quadeer AA, McKay MR. Preliminary identification of potential vaccine targets for the COVID-19 coronavirus (SARSCoV-2) based on SARS-CoV immunological studies. Viruses 2020;12:254.
6 Pan Y, Zhang D, Yang P, et al. Viral load of SARS-CoV-2 in clinical samples. Lancet Infect Dis 2020;20:411-2.

7 World Health Organization. Population-based age-stratified seroepidemiological investigation protocol for coronavirus 2019 (COVID-19) infection, 2020. Available: https://www.who.int/ publications/i/item/WHO-2019-nCoV-Seroepidemiology-2020.2. [Accessed 5 Jul 2020].

8 Sood N, Simon P, Ebner P, et al. Seroprevalence of SARS-CoV-2specific antibodies among adults in Los Angeles County, California, on April 10-11, 2020. JAMA 2020;323:2425-7.

9 Dimeglio C, Loubes J-M, Deporte B, et al. The SARS-CoV-2 seroprevalence is the key factor for deconfinement in France. $J$ Infect 2020;81:318-56.

10 Song SK, Lee DH, Nam JH, et al. IgG seroprevalence of COVID-19 among individuals without a history of the coronavirus disease infection in Daegu, Korea. J Korean Med Sci 2020;35:e269.

11 Noh JY, Seo YB, Yoon JG, et al. Seroprevalence of anti-SARS-CoV-2 antibodies among outpatients in southwestern Seoul, Korea. $J$ Korean Med Sci 2020;35:e311.

12 Pourhoseingholi MA, Vahedi M, Rahimzadeh M. Sample size calculation in medical studies. Gastroenterol Hepatol Bed Bench 2013;6:14-17.

13 Bendavid E, Mulaney B, Sood N. COVID-19 antibody seroprevalence in Santa Clara County, California. medRxiv 2020.

14 Roche. Elecsys ${ }^{\circledR}$ Anti-SARS-CoV-2, 2020. Available: http:// iagnostics.roche.com/global/en/products/params/elecsys-anti-sarscov-2.html [Accessed 11 Aug 2020].

15 Havers FP, Reed C, Lim T, et al. Seroprevalence of antibodies to SARS-CoV-2 in 10 sites in the United States, March 23-May 12, 2020. JAMA Intern Med 2020. doi:10.1001/ jamainternmed.2020.4130. [Epub ahead of print: 21 Jul 2020].

16 Pollán M, Pérez-Gómez B, Pastor-Barriuso R, et al. Prevalence of SARS-CoV-2 in Spain (ENE-COVID): a nationwide, population-based seroepidemiological study. Lancet 2020;396:535-44.

17 Bogogiannidou Z, Vontas A, Dadouli K, et al. Repeated leftover serosurvey of SARS-CoV-2 IgG antibodies, Greece, March and April 2020. Euro Surveill 2020;25:2001369.

18 Hallal PC, Hartwig FP, Horta BL, et al. SARS-CoV-2 antibody prevalence in Brazil: results from two successive nationwide serological household surveys. Lancet Glob Health 2020;8:e1390-8.

19 Xu X, Sun J, Nie S, et al. Seroprevalence of immunoglobulin M and G antibodies against SARS-CoV-2 in China. Nat Med 2020;26:1193-5.

20 Cheng MP, Yansouni CP, Basta NE, et al. Serodiagnostics for Severe Acute Respiratory Syndrome-Related Coronavirus 2 : A Narrative Review. Ann Intern Med 2020;173:450-60.

21 Korea Disease Control and Prevention Agency. Updates on COVID-19 in Republic of Korea (as of 14 September), 2020. Available: http://ncov.mohw.go.kr/en/ tcmBoardView.do?brdld=12\&brdGubun=125\&dataGub un=\&ncvContSeq=3692\&contSeq=3692\&board id=\&gubun= [Accessed 20 Oct 2020].

22 Stringhini S, Wisniak A, Piumatti G, et al. Seroprevalence of antiSARS-CoV-2 IgG antibodies in Geneva, Switzerland (SEROCoVPOP): a population-based study. Lancet 2020;396:313-9.

23 Xie X, Zhong Z, Zhao W, et al. Chest CT for typical coronavirus disease 2019 (COVID-19) pneumonia: relationship to negative RTPCR testing. Radiology 2020;296:E41-5.

24 Zou L, Ruan F, Huang M, et al. SARS-CoV-2 viral load in upper respiratory specimens of infected patients. $N$ Engl J Med 2020;382:1177-9.

25 Guo L, Ren L, Yang S, et al. Profiling early humoral response to diagnose novel coronavirus disease (COVID-19). Clin Infect Dis 2020;71:778-85.

26 Zhao J, Yuan Q, Wang $\mathrm{H}$, et al. Antibody responses to SARS-CoV-2 in patients with novel coronavirus disease 2019. Clin Infect Dis 2020;71:2027-34.

27 Wu F, Wang A, Liu M, et al. Neutralizing antibody responses to SARS-CoV-2 in a COVID-19 recovered patient cohort and their implications. medRxiv 2020.

28 Long QX, Deng HJ, Chen J. Antibody responses to SARS-CoV-2 in COVID-19 patients: the perspective application of serological tests in clinical practice. medRxiv 2020. 